\title{
Interactive Informatics on Internet Infrastructure
}

\author{
F. Zhao, V. R. Vemuri, S. F. Wu \\ Department of Computer Science \\ University of California, Davis \\ \{fanzhao, rvemuri, sfwu\}@ucdavis.edu
}

\author{
F. Xue, S. J. B. Yoo \\ Department of Electrical and Computer Engineering \\ University of California, Davis \\ \{xue, yoo\}@ece.ucdavis.edu
}

\begin{abstract}
We present the design and evaluation of I4, a network infrastructure that enables information exchange and collaboration among different domains. I4 can help address problems, such as defending against the unwanted traffic as well as diagnosing the network. We present the Distributed Denialof-Service (DDoS) attack as an example to demonstrate the advantages of I4. Simulation results show that I4 can significantly reduce the amount of DDoS attack packets and dramatically improve the quality of services received by legitimate users. Our design provides attractive properties, such as incremental deployment as well as incentives for such deployment etc.
\end{abstract}

\section{INTRODUCTION}

The current Internet infrastructure follows the end-to-end $(e 2 e)$ principle, which states that functionality should be placed as close to the network edges as possible, keeping the network core focused on the task of routing packets. Thus an ISP domain simply forwards all the traffic to its customers ${ }^{1}$ with "best efforts" and the customer domains passively receive all the traffic arriving at their links.

Indeed, the $e 2 e$ principle simplifies the Internet design and contributes greatly to the success of the Internet witnessed during the last two decades. However, in many scenarios, the domains, either ISPs or customers, would benefit from additional "meta-information" (or "information", in short) related to the current ongoing $e 2 e$ flows. For example, the ISP usually has no knowledge to identify unwanted traffic, such as DDoS attack packets, destined for a different domain; on the contrary, as the actual recipient, the customer domain can identify the offending or unwelcome packets based on its rich capability of intrusion detection or its preferences. If this information, whether certain traffic is wanted or not, is available, the ISP would take actions to eliminate those harmful or useless packets. This not only reduces the traffic load inside the ISP domain, but also prevents the resources in the customer domains from being wasted. Unfortunately the current Internet infrastructure does not provide any means for different domains to exchange such useful information.

This limitation has motivated many proposed solutions ${ }^{2}$. While originally proposed to identify the real path taken by spoofed DDoS attack packets, iTrace/traceback mechanisms [1] [2] [3] [4] [5] enable the ISP domains to propagate the (path) information to victim domains. In ACC/Pushback [11]

\footnotetext{
${ }^{1}$ Generally speaking, given the path taken by a traffic flow, denoted by $S \rightarrow D_{0} \rightarrow \ldots \rightarrow D_{n} \rightarrow D, S$ and $D$ are the customer domains, and $D_{0}, \ldots, D_{n}$ are the ISP domains.

${ }^{2}$ Please refer to section VIII for a complete survey on the related works.
}

[12] and Route Throttles [10], the information about the aggregates received and server load is sent back to certain upstream routers where the high-volume aggregates are rate-limited. I3 [19] proposed to decouple the act of sending from the act of receiving; thus the domain needs to insert the binding between its identity and location into the network. SIFF [8] and TVA [17] proposed an end-host capability control mechanism to limit the DDoS attack: the capability is generated by the ISP domains, piggybacked to the recipient and further forwarded to the sender as an explicit authorization. Another example is active network [18] where executable code is sent to the routers in the "black-boxed" Internet core.

All of these pioneering works indicate the need to improve the Internet infrastructure, and each of them has its own merit. Yet we argue that none of them has given a complete tool box for tackling all the problems in today's Internet; moreover, there is still significant space for improvements even with the latest proposals.

In this paper, we propose Internet Information Interaction Infrastructure (I4) to enable the information exchange among domains. It leverages on the most common form of communication, unicast. Our contributions are multifold: First, we present a generic inter-domain information exchange framework within which a large range of challenging problems, such as DDoS attacks, worm, and network failure etc, can be tackled. Second, we present the detailed design of this infrastructure, and demonstrate the effectiveness of information exchange to resist the DDoS attack with extensive experiments and simulations. Third, we describe many accompanying algorithms and ideas, such as weight-based resource allocation and scheduling, estimation by adaptive sampling and Mean Square Estimation (MSE), and BGP-based key distribution mechanism, as possible improvements over previous proposals.

We design $\mathrm{I} 4$ to be practical in the following key aspects. First, the design of I4 strongly incents both ISP domains and customer domains to deploy. Second, I4 supports the incremental deployment that allows the participating domains to have the immediate benefits. Third, we examine many related factors to make the information exchange procedure efficient, robust and secure.

This paper is organized as follows. Section II describes the architecture of I4. Section III describes the detailed information interaction procedure in the case of DDoS attacks. In section IV we propose approaches to extract accurate knowledge 


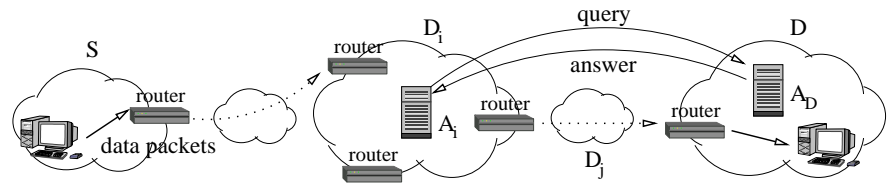

Fig. 1. The I4 agent in $D_{i}$, denoted by $A_{i}$, observes some packets in the $e 2 e$ communication between $S$ and $D . A_{i}$ generates a query and sends to the I4 agent, $A_{D}$, in $D$. When this query arrives at $D, A_{D}$ generates an answer with the help of local Intrusion Detection System (IDS) and other knowledge, and returns back to $A_{i}$. Finally $A_{i}$ will make decisions based on the content of the answer and its local policy.

from the information exchanged, even if the information is incomplete. Section V studies several procedures to throttle the DDoS attack traffic by applying the extracted knowledge and presents the NS-2 simulation results. In the following sections we summarize the advantages of I4, discuss related issues and present a survey of related works.

\section{ThE ARCHITECTURE OF I4}

\section{A. Overview}

Conceptually, each Autonomous System ${ }^{3}$ (AS) in the Internet supporting I4 has one I4 agent responsible for the task of information interaction in this domain (see Fig. 1). Generally speaking, when one agent in the Internet observes a piece of original "information", e.g. one or a sequence of packets, it generates a query regarding this piece of information, and sends this query, usually together with some additional information, to another agent that is responsible for interpreting the query and providing an answer. Finally the agent that sent the query will take actions based on the content of the answer received. This kind of information exchange is termed the "pull" mode. (From the perspective of the initiator, the last event in the information exchange procedure ${ }^{4}$ is to pull the information from the responder.)

One of the necessary conditions for information exchange is the availability of the responder's identity or location. However, due to asymmetric routing, the customer domain usually has no idea about which ISP domain currently forwards the traffic coming toward itself 5 . "Pull" mode may be the most suitable way for information exchange in this situation: the ISP domain attaches its location/identity information in the query so that the customer domain knows where to send the answer back.

In other scenarios, the initiator may already know the identity/location of the responder, e.g. via previous information exchange or a service agreement established through some additional channel. If it is the case, the query is not a necessary condition for an answer to be triggered. This kind of information exchange is referred as "push" mode because the

\footnotetext{
${ }^{3}$ In this paper, "AS" is used exchangeably with "domain".

${ }^{4}$ Precisely speaking, all information exchange events are correlated more or less in the long term, and all of them constitute one large procedure that lasts through the lifetime of one agent. In this paper, we abstract a set of temporally and content correlated events as one information exchange procedure.

${ }^{5}$ Instead, the ISP domain can implicitly infer how to reach the agent in the customer domain from the destination IP address in the packets forwarded.
}

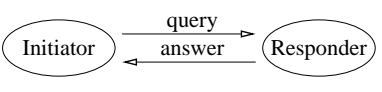

(a) Pull mode

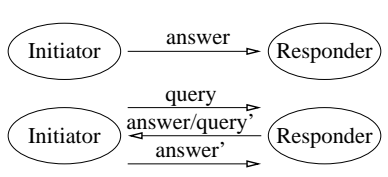

(b) Push mode
Fig. 2. Different information exchange modes

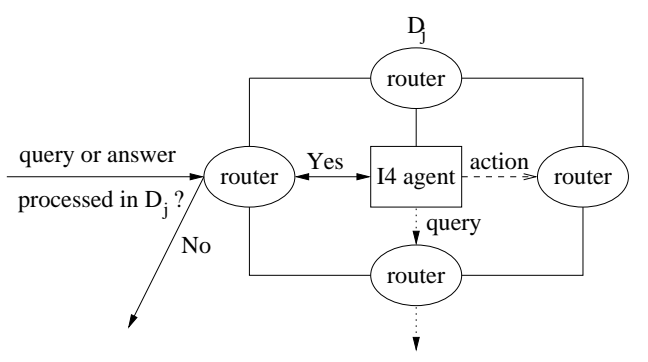

Fig. 3. Interactions between routers and an I4 agent

initiator pushes the information to the responder. Fig. 2 shows the procedures of both modes. Note that a more complicated "push" mode may require some preliminary communication to fulfill certain prerequisites for the final "push". Fig. 2(b) shows such an example.

To exchange information, query and answer are either piggybacked in the originally observed data packet or conveyed by an out-of-band message. Different choices result in different tradeoffs between overhead and flexibility. Either way, query and answer are usually carried by unicast IP packets that are routed by Internet standard routing protocol, i.e. BGP.

\section{B. Intra-AS issues}

Due to scalability consideration, the function of the I4 agent may be implemented in as few as just one node inside one AS. In order to exert its capability, the I4 agent has to collaborate with other entities, such as IDS/IPS and routers. The necessary network and intra-domain routing configurations must be set up in advance in order for them to communicate with each other. Moreover, the security association between I4 agent and other entities is established and the time-synchronization is maintained. These requirements are reasonable because they are under the same administration domain.

A router plays an important role in the information exchange. First, it selects some data packets based on certain criteria and forwards them to the I4 agent so that a query can be generated. Second, query and answer are forwarded by routers to the corresponding I4 agents based on their Forwarding Information Bases (FIBs). In addition, during the transmission a router in the I4 domain checks whether an incoming query or answer should be processed in this domain; if so, the router forwards it to the local I4 agent. Third, after an I4 agent receives the answer and decides the actions to take, it communicates with routers where the decisions will take effect. Fig. 3 shows such interactions between routers and an I4 agent. 


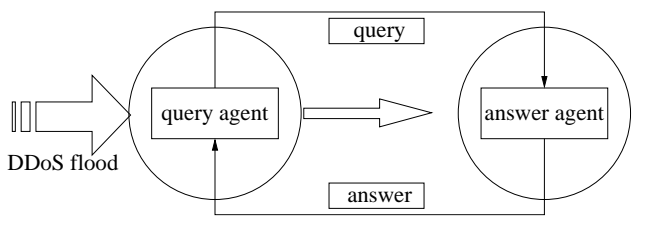

Fig. 4. Feedback model

It is also possible, especially in one large AS, that there are multiple I4 agents set up for the purpose of faulttolerance, load-balancing, etc. In order to achieve scalability when enabling the inter-agent communication, these I4 agents can be organized in the same way as route-reflection and consolidation in BGP. The details of such kinds of interagent communication protocol and hierarchy organization are beyond the scope of this paper.

\section{INFORMATION INTERACTION IN THE CASE OF DDOS ATTACKS}

\section{A. Overview}

DDoS attacks are deemed as the first-order threat in the Internet. The infamous attack in February 2000 caused major Internet portals such as Yahoo, eBay and E*Trade to shut down. Despite the lack of media attention after that, DDoS attacks are even more severe and prevalent in the Internet. Today the binary codes or even the complete packages of DDoS attack tools are readily available and do not require sophisticated knowledge to launch. In a previous paper [14], the authors reported a surprisingly huge number of DDoS attacks observed in everyday traffic.

We apply the "pull" information exchange mode in the case of DDoS attack. We call the agent in the ISP domain "query agent" and the agent in the customer domain (i.e. the target of attackers) "answer agent". As shown in Fig. 4, the information exchange procedure can be formulated as a feedback model: the query is a signal to the customer domain while the answer serves as a feedback to the ISP domain; after several times of exchanges, this feedback mechanism would make the whole system converge to an equilibrium state. In the following we present detailed packet formats and information interaction procedures.

\section{B. Query}

1) Query generation: To generate a query, routers in the ISP domain randomly select a data packet with a certain probability, $\mathrm{Pr}$, and forwards this selected packet ${ }^{6}$ to the query agent. We propose to piggyback the query in the selected data packet because an out-of-band query message results in more overheads. Although this may cause the fragmentation if Maximum Transmission Unit (MTU) is exceeded, we argue that the adverse impacts are little because: 1) the attacker tends to use smaller packets in order to exhaust the router resources

\footnotetext{
${ }^{6}$ Additional information, such as the IP address or the identity of the router, the interface where this selected data packet arrives or departs, and the local time, may be forwarded to the query agent as well.
}

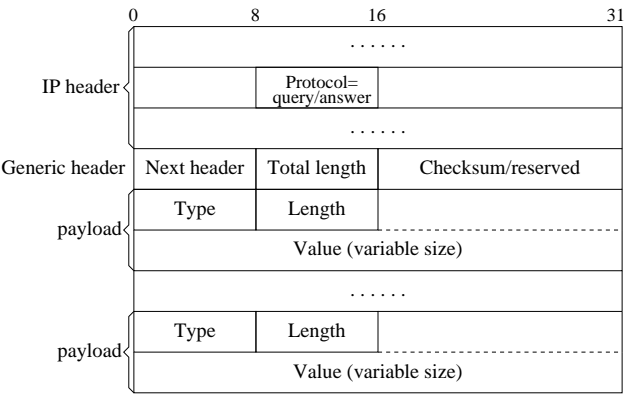

Fig. 5. The format of I4 query or answer

TABLE I

PAYLOADS IN THE QUERY

\begin{tabular}{|c|c|c|}
\hline Payload Type & Length & Description \\
\hline Query agent & 4 bytes & $\begin{array}{l}\text { the query agent's IP address at which the } \\
\text { answer will be received }\end{array}$ \\
\hline Router ID & 2 bytes & $\begin{array}{l}\text { to identify the router that selects this data } \\
\text { packet }\end{array}$ \\
\hline Interface ID & 1 bytes & $\begin{array}{l}\text { to identify the interface where the } \\
\text { selected data packet arrives or departs }\end{array}$ \\
\hline Timestamp & 4 bytes & $\begin{array}{l}\text { the local time when the router selects this } \\
\text { data packet }\end{array}$ \\
\hline Sequence No. & 4 bytes & a counter maintained by the query agent \\
\hline Cookie & 16 bytes & $\begin{array}{l}\text { a random number generated for stateless } \\
\text { verification }\end{array}$ \\
\hline
\end{tabular}

to the maximum level; 2) the attack packet itself is small in many challenging DDoS attacks, such as SYN flooding and setup channel flooding [17]; 3) the query is piggybacked in the first fragmentation; thus once an attack fragmentation is identified, the whole packet does not have to be re-assembled.

We propose a new type of IP protocol, called "query", which is placed in the protocol field in the original IP header. The query starts with a generic header where the next header field indicates the type of next header, either an upper transport protocol or another query. Each payload follows the TypeLength-Value (TLV) format as some payloads are optional or of variable size. See Fig. 5 for more details.

The value of $\operatorname{Pr}$ should be carefully chosen in order to strike a balance between the overhead of IP packet processing and the amount of information exchanged. Furthermore, a router could select the data packet from different aggregates ${ }^{7}$ [11] with different probabilities, thus it can spend more resources for some aggregates of special interests, e.g. those destined for one preferred customer domain whose intention of reception is distributed proactively or reactively [5].

2) Query payloads: Table I lists the descriptions and the suggested lengths of payloads appearing in the query. Note that in practice, there may be more efficient way to represent these payloads. We briefly discuss below the use of these payloads. (See section IV for more details.)

- "Router ID" and "Interface ID": The administrator can assign unique numbers to routers and their interfaces.

${ }^{7}$ The traffic can be separated into aggregates based on the interfaces that the packets arrive at or depart from, destination IP address or prefix, or the output of some clustering algorithm. 


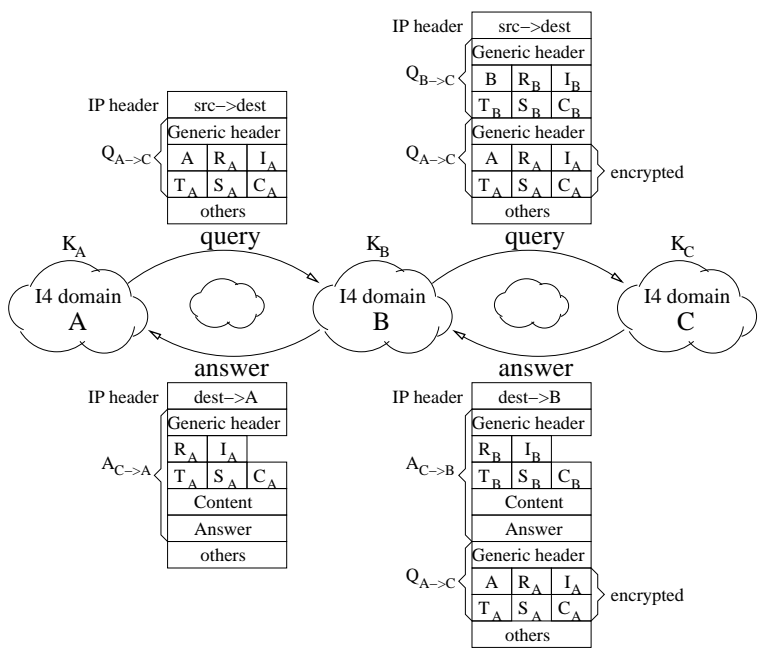

Fig. 6. Information exchange procedure: $R_{A}, I_{A}, T_{A}, S_{A}$ and $C_{A}$ denote "Router ID", "Interface ID", "Timestamp", "Sequence number" and "Cookie" generated by $A$ respectively. The payloads generated by $B$ are denoted in the same way. Note that $\mathrm{C}$ copies $R_{B}, I_{B}, T_{B}, S_{B}$ and $C_{B}$ from the received query $Q_{B \rightarrow C}$ into the answer message $A_{C \rightarrow B}$.

These two payloads identify the origin of the information (either a query or answer) received by an I4 agent. Also later a query agent can know where the corresponding knowledge should be distributed.

- "Timestamp": It allows a query agent to learn the temporal property of the information received. This payload together with "Router id" and "Interface id" is provided by routers to the query agent.

- "Sequence number": To resist the replay attack, this payload contains the current value of a counter incremented by one when a query is sent.

- "Cookie": It allows the query agent to statelessly verify that the received answer is indeed in response to a query generated by itself earlier. This payload contains the output of the following formula:

$$
H(K, \text { Query agent } \| \text { destIP } \| \text { other payloads })
$$

where $K$ is the secret key generated by the query agent and $H$ is a secure hash function.

In the case of a DDoS attack, the query is like a question to the answer agent: Is this selected data packet good or bad?

3) Query transmission: As the destination IP address is not changed, the query is forwarded to the same destination domain as the original data packet selected. Fig. 6 shows the procedure of query transmission. $A$ generates a query, $Q_{A \rightarrow C}$, to $C$. If there is an I4 domain, say, $B$ on the route from $A$ to $C$, it treats this received query just like a selected data packet: $B$ first encrypts $Q_{A \rightarrow C}$ (except the generic header) with its secret key, $K_{B}$, and then inserts its own query $Q_{B \rightarrow C}$ in between the IP header and $Q_{A \rightarrow C}$. Note that the cookie payload in $Q_{B \rightarrow C}$ also covers the encrypted portion of $Q_{A \rightarrow C}$. Finally this query will arrive at the destination domain $C$.
TABLE II

ADDITIONAL PAYLOADS IN THE ANSWER MESSAGE

\begin{tabular}{|c|c|l|}
\hline Payload Type & Length & Description \\
\hline Content & variable & $\begin{array}{l}\text { IP header and the partial upper layer payloads } \\
\text { in the selected data packet }\end{array}$ \\
\hline Answer & 1 byte & the evaluation result of the selected data packet \\
\hline Signature & variable & the signature of good or bad traffic \\
\hline Duration & 4 bytes & the validity period of the supplied signature \\
\hline
\end{tabular}

TABLE III

Possible VAlues AND MEANINGS OF THE ANSWER PAYLOAD

\begin{tabular}{|c|l|}
\hline Value & Description about the selected data packet \\
\hline$(00)_{16} \leq y \leq(64)_{16}$ & the prob. as a bad packet is $y \%$ \\
$(32)_{16}$ & unknown, 50\% as a bad packet \\
$(00)_{16}$ & a good packet, 0\% as a bad packet \\
$(64)_{16}$ & a bad packet, 100\% as a bad packet \\
\hline
\end{tabular}

\section{Answer}

1) Answer message generation: As the actual recipient, the customer domain is the most appropriate one to answer whether this selected data packet is good or bad. In addition, with the help of local IDS/IPS, it is indeed capable to provide an accurate answer ${ }^{8}$.

We use an out-of-band message to carry the answer because some $e 2 e$ communication is unidirectional. Similarly, we design a new type of IP protocol, called "answer", , shown in Fig. 5. In the answer message, the source IP address is the destination IP address in the received query, and the destination IP address is the value of "Query agent" payload, i.e. the IP address of the query agent. The answer agent has various strategies to respond to queries; for example, it may cluster the answers to a set of queries in one answer message in order to reduce the overhead.

2) Answer message payloads: All the payloads (except the first "Query agent" payload in cleartext, but including the following encrypted portions) in the received query should be copied into the generated answer message. In addition, as shown in Table II the following four new payloads may appear in the answer message.

- "Content": This allows the query agent to correlate the received answer with the originally selected data packet.

- "Answer": This contains the evaluation result, namely, the probability that the selected data packet is bad. Table III shows the possible values in this payload. The probability as a good packet can be calculated easily.

- "Signature": When combining with "Answer" payload, this optional payload indicates the signature of the good or bad traffic represented by the header and/or the partial data payload, thus the query agent could install some filters based on the received signature in the appropriate routers.

- "Duration": This indicates the validity period of a provided signature. Note that the query agent may indepen-

${ }^{8}$ Indeed, the partial path information carried in the query may help identify the attack packet.

${ }^{9}$ The numeric values for the types of "query" and "answer" protocols will be assigned by IANA. 
dently set up the lifetime for the received signature rather than based on this payload.

3) Answer message transmission: Fig. 6 shows the procedure of answer message transmission. When $B$ receives an answer message from $C$, it first checks if this is a replayed message by examining the "Sequence number" payload, $S_{B}$, just like the anti-replay window mechanism in IPSec. Then $B$ reconstructs the cookie based on Equation 1 with IP addresses and related payloads as inputs. Note that the order of the source IP address and the destination IP address in the received answer message should be reversed when calculating the cookie. $B$ accepts this answer message if the output matches with the "Cookie" payload, $C_{B}$, received or simply discards it otherwise. After the validation, $B$ may update its knowledge and take further actions based on the received information. Furthermore, $B$ decrypts the first encrypted query, $Q_{A \rightarrow C}$ in this example and constructs an answer message for $A$ based on, e.g. the "Content" payload and the "answer" payload, generated by $C$. When $A$ receives $A_{C \rightarrow A}$ from $B$, it follows the same procedure to verify the received answer message and takes the information into consideration if succeed.

\section{Discussion}

Our information exchange protocol is efficient and lightweight because it does not maintain the connectionoriented states like in TCP. "Cookie" payload enables the query agent to statelessly verify the received answer message. It is computationally impossible for an attacker to forge a valid cookie without the knowledge of the secret key, $K$. Although it is still vulnerable to Man-in-Middle attack, it does not introduce any new threat. See section VII for more discussion on security issues.

Query and answer may be lost or reordered during the transmission. With the anti-replay sliding window and the stateless verification, our protocol is robust against packet reordering. Moreover, "Timestamp" payload allows the query agent to apply the received information properly, especially when an answer experiences the long transmission delay. Finally as we will show in section IV, adaptive sampling can tolerate I4 packet loss.

\section{KNOWLEDGE EXACTION FROM INFORMATION INTERACTION}

From the information exchanged, the customer domain and the ISP domain can extract the knowledge about aggregates, as we shall show below. An aggregate can be denoted by $\left\langle I, R_{i d}, I_{i d}, C\right\rangle$ if it is destined for a customer domain $C$ and arrives at an interface $I_{i d}$ of one particular router $R_{i d}$ in the ISP domain $I$. Each element in this vector can be either 0 or represent a particular domain, router or interface. We use Fig. 6 to illustrate our method. Table IV summarizes the notation used in this section.

\section{A. Knowledge}

1) The arrival rate of traffic: Every domain can estimate the arrival rate of aggregates arriving at their local links. We
TABLE IV

\section{NOTATION}

\begin{tabular}{|c|l|}
\hline$T$ & $\begin{array}{l}\text { the length of the time period during which a percentage is } \\
\text { measured by counting the received answer messages } \\
\text { the number of answer messages regarding this aggregate } \\
\text { received during } T \\
N_{A}\end{array}$ \\
$N_{A}^{b}$ & $\begin{array}{l}\text { the number of answer messages with negative evaluation } \\
\text { results regarding this aggregate received during } T \\
\text { the percentage of bad packets of one aggregate }\end{array}$ \\
$P_{c}$
\end{tabular}

adopt the following formula from [11]:

$$
R_{\text {new }}=\left(1-e^{-\frac{t}{k}}\right) R_{\text {current }}+e^{-\frac{t}{k}} R_{\text {old }}
$$

where $R_{\text {current }}=\frac{l}{t}, t$ is the inter-packet interval, $k$ is a constant, e.g. $k=2$ and $l$ is the average length of data packets.

Moreover, with "Query agent", "Router ID" and "Interface ID" payloads in the received query, the customer domain $C$ can estimate the arrival rate of aggregates forwarded by one remote ISP domain, $B$, because each query is a randomly selected sample of the traffic. For example, if within $T$ seconds the number of queries that $C$ receives from $B$ is $N_{Q, B}$ and the probability of query generation is $\mathrm{Pr}$, the rate of the aggregate $\langle B, 0,0, C\rangle$ is $\frac{N_{Q, B}}{\operatorname{Pr} * T}$ packets per second.

2) The percentage of bad packets: $C$ can further estimate the percentage of bad packets, denoted by $P c$, of the aggregates forwarded by $B$. For example, assume within $T$ seconds the number of queries from $B$ received by $C$ is $N_{Q}-1$ and $C$ generates the answers $\left\{A_{0}, \ldots, A_{N_{Q}-1}\right\}$. Recall that $A_{i}, 0 \leq$ $i \leq N_{Q}-1$, contains the probability that a selected data packet is bad. Then $P c$ of the aggregate $\langle B, 0,0, C\rangle$ during this time period is estimated as $\frac{\sum A_{i}}{N_{Q}}$ where $0 \leq i \leq N_{Q}-1$. With additional "Router ID" and "Interface ID", $C$ can estimate $P c$ of even "smaller" aggregates.

Similarly, $B$ can estimate $P c$ of the aggregate $\left\langle B, R_{i d}, I_{i d}, C\right\rangle$ based on the answer messages received from $C$. Assume that within $T$ seconds, the answer messages with the router $\mathrm{id}, R_{i d}$, and the interface $\mathrm{id}, I_{i d}$, received from $C$ are $\left\{A_{0}, \ldots, A_{N_{A}-1}\right\}$. Then $P c$ of the aggregate $\left\langle B, R_{i d}, I_{i d}, C\right\rangle$ during this time period is $\frac{N_{A}^{b}}{N_{A}}=\frac{\Sigma A_{i}}{N_{A}}$, where $0 \leq i \leq N_{A}-1$.

Note that we assume that each data packet in aggregates is discrete when calculating $P c$. In fact, if one selected data packet belongs to one session, we can label all the packets in this session based on the answer regarding this selected data packet.

\section{B. More about percentage estimation}

A smaller query generation probability can reduce the processing overhead and traffic load, however it results in inaccurate percentage estimation. The previous subsection presents a basic approach to estimate the percentage of bad packets. Fig. 7 gives a simple result of implementing a "sample and hold" strategy which is nothing but a zero-order interpolation. From this figure we can see that when the probability of query generation becomes smaller, the error in estimation becomes bigger. However even when $\operatorname{Pr}=0.005$, the estimation is still 


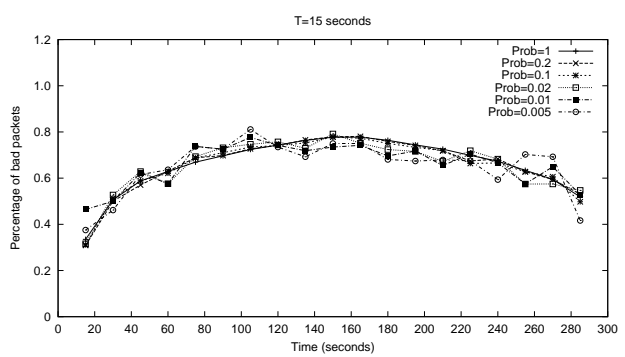

Fig. 7. Percentage of bad packets when $\mathrm{T}=15$

close to the real data. We will apply these parameters in the simulation of rate-limiting DDoS attack traffic in section V.

In the future, we propose to study this issue further in depth. For example, two questions that need to be answered are: 1) how to adapt to the traffic dynamics and adjust the corresponding parameters to provide an accurate estimation? 2) how to apply the estimated percentage to the current incoming traffic and promptly adapt to the sudden changes?

To address the first question, we intend to apply the adaptive sampling method where the sampling procedure depends on the dynamic properties of the variables of interest observed during the past. Thus it gives flexibility to change the sampling plan during the course of the survey in response to observed patterns.

To address the second question, we can analyze the trend from the last $m$ measured percentages, then estimate $P c$ in the near future, for example, using Linear Mean Square Estimation (LMSE) and ARMA. It is also interesting to evaluate these approaches with the real DDoS attack trace in the realistic topology.

\section{EXAmples of Collaborative Defense AGAinst DDOS ATTACKS}

I4 is capable to address any kind of unwanted traffic. If a "signature" is available, such as existing TCP sessions, nonspoofing packet flooding and worm traffic, a filter based on the answer message can be installed in the upstream domain. With the knowledge described in section IV, I4 is even more powerful in that it can address more challenging attacks, such as spoofing attack ${ }^{10}$ and initial requests flooding [17]. In this section, we assume a general form of the DDoS attack where a "signature" is not available.

Our observation is that during a DDoS attack, bad traffic contends limited resources, such as the packet scheduling, the link bandwidth etc, with the good traffic. However, currently the router cannot distinguish the "good" from the "bad". I4 precisely addresses this limitation. In the following we show what information and how exchange of this information would help mitigate the effects of DDoS attacks.

\footnotetext{
${ }^{10}$ Due to the lack of IP address accountability, an attacker can easily conceal his/her location(s). Moreover a header or content based filter may cause the bilateral damage.
}

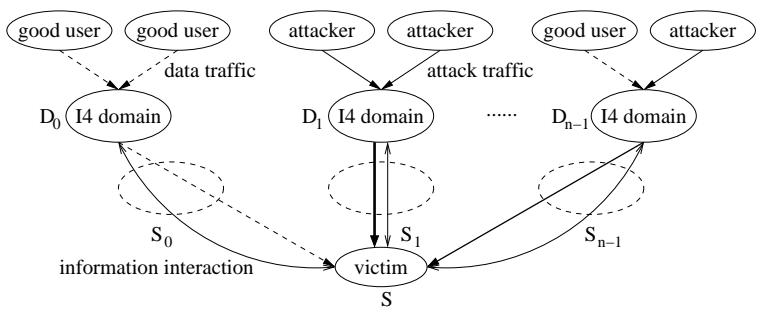

Fig. 8. Max-Min Fair Server-Centric Router Throttles

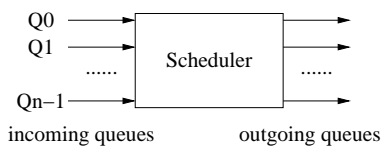

Fig. 9. Weighted queue scheduling mechanism

\section{A. Differentiated server load balancing mechanism}

Reference [10] proposed that during the DDoS attack a server (the victim) indicates the load it desires to specific upstream routers, then routers drop the excess traffic to the server. For example, assume that there are $n$ upstream domains, $\left\{D_{0}, D_{1}, \ldots, D_{n-1}\right\}$, forwarding the traffic to one victim domain, $V . V$ splits its total server load, $S$, into $\left\{S_{0}, S_{1}, \ldots, S_{n-1}\right\}$ and then indicates this information to the corresponding upstream domains, $D_{i}$. However in [10] $V$ does not split its server load optimally. As we described above, with the information exchange the victim domain can now estimate the volume of traffic forwarded by each upstream domain $D_{i}$ and which upstream domain forwards the "better" traffic in terms of the percentage of bad packets within. Thus $V$ can assign the larger workloads to those $D_{i}$ forwarding a lower percentage of bad packets, which makes the victim domain not only receive the appropriate amount of traffic without exceeding its capacity, but also serve more "good" packets from the legitimate users.

\section{B. Weighted queue scheduling mechanism}

In this section we propose a weighted queue scheduling mechanism that schedules packet forwarding from one incoming queue to one outgoing queue based on the weight assigned to the incoming queue.

1) Description: Given a router with $n$ queues (Usually each queue has the same characteristics, such as bandwidth and delay.), $\left\{Q_{0}, Q_{1}, \ldots, Q_{n-1}\right\}$, let the percentage of bad packets in each queue $Q_{i}$ be $p_{i}$. Each queue $Q_{i}$ is assigned a weight, $w_{i}=f\left(1-p_{i}\right)$, where $f()$ is an ascending function or simply $f(x)=x$.

In the classical "Round Robin" scheduling mechanism, each $Q_{i}$ is scheduled with the same weight and then the percentage of bad packets forwarded by this router is equal to $Y=\frac{\Sigma p_{i}}{n}$. In this proposed mechanism, the ratio between the number of packets forwarded from $Q_{i}$ and the total number of packets forwarded by the router is equal to $\frac{w_{i}}{\Sigma w_{i}}$ and the percentage of bad packets forwarded by the router is equal to $X=\frac{\Sigma p_{i} * w_{i}}{\Sigma w_{i}}$ where $i=0,1, \ldots, n-1$. It can be easily proven that $X \geq Y$ 


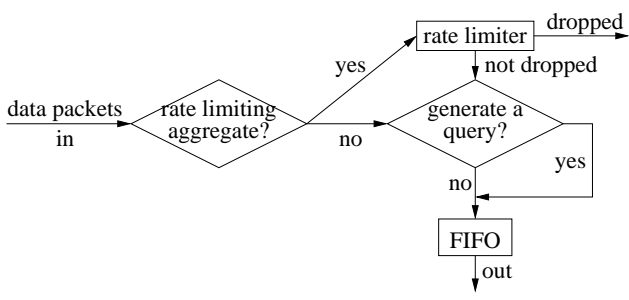

Fig. 10. Rate limiting procedure

and $X=Y$ if and only if $p_{0}=p_{1}=\ldots=p_{k}$. Thus the weighted queue scheduling mechanism is better than or as good as the "Round Robin" mechanism in terms of the overall percentage of bad packets forwarded.

By assigning a higher weight to the queue containing a lower percentage of bad packets, the router in the ISP domain spends more resources in forwarding the packets from these "good" queues. Thus the queue with the higher percentage of bad packets tends to become full and eventually more bad packets are dropped.

2) Discussion: The proposed mechanism is based on the preferential scheduling of the shared resources among interfaces. Although the modern "carrier-class" router starts to have more and more parallelism built in, there might still exist many central resources shared among linecards. Moreover there are still a lot of legacy routers, for example, without dedicated CPU or memory for each linecard, or without full-mesh cross-bar. As it is these slower routers that are more likely congested during the DDoS attack, this proposed mechanism could significantly improve the performance of good sessions if implemented in these bottlenecks.

\section{Weighted aggregate-scheduling mechanism}

We propose another scheduling mechanism based on the weight of aggregates inside each queue.

1) Overview: Assume that there are $n$ aggregates in one unidirectional queue, $Q,\left\{A_{0}, A_{1}, \ldots, A_{n-1}\right\}$. The arrival rate of $A_{i}$ is $R_{i} \mathrm{pkt} / \mathrm{sec}$ or $B_{i} \mathrm{Mb} / \mathrm{sec}$, thus the total arrival rate of all aggregates is $\Sigma B_{i} \mathrm{Mb} / \mathrm{sec}$. Also we assume that the probability to generate an I4 query from the packets arriving at $\mathrm{Q}$ is $P$ and the bandwidth of $Q$ is $B \mathrm{Mb} / \mathrm{sec}$.

When the total arrival rate, $\Sigma B_{i}$, is larger than the predefined threshold, for example, $c * B$ where $c$ is a constant factor, the router starts to rate-limit the incoming traffic in this queue. The total of excess traffic to be dropped is $\Sigma B_{i}-c * B$. Fig. 10 shows the procedure of processing an incoming packet in 14 queue during the congestion. The router checks whether this packet belongs to an aggregate to be rate-limited. If yes, the packet is forwarded to a rate-limiter module that determines whether this packet should be dropped. If not, the packet is forwarded to a query generation module that generates a query based on this packet with the probability $P$.

2) Rate-limiting algorithms: Algorithm 1 shows the pseudo code of greedy rate-limiting algorithm implemented in our simulation.
Algorithm 1 Greedy rate-limiting algorithm

Sort the aggregates in the descending order of the percentage of bad packets, for exmaple, $\left\{A_{i_{0}}, A_{i_{1}}, \ldots, A_{i_{n}}\right\}$.

$j \Leftarrow 0, E \Leftarrow \Sigma_{k=0}^{n} R_{i_{k}}-B$ where $R_{i_{k}}$ is the arrival rate of $A_{i_{k}}$ and

$B$ is the link bandwidth.

Given an incoming packet, $p k t$, for each aggregate, $A_{i_{j}}$, if $p k t \in A_{i_{j}}$

pkt is dropped with the probability, $\min \left\{E / R_{i_{j}}, 1\right\}$, and exit the loop.

else if $R_{i_{j}}>E$, then pkt is forwarded and exit the loop. else $E \Leftarrow E-A_{i_{j}}, j \Leftarrow j+1$

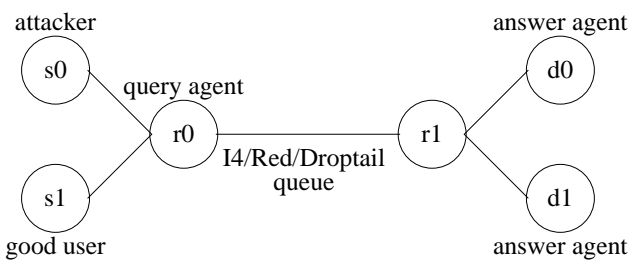

Fig. 11. The simulation topology

Besides, other rate-limiting algorithms, such as tokenbucket rate-limiting algorithm [11] and weighted rate-limiting algorithm as described in section $\mathrm{V}-\mathrm{B}$, are also possible. Compared with ACC/Pushback [11], our proposal is more general because the high-bandwidth aggregate may not be attack traffic always and it may depend on the positions of routers in the Internet. With the information learned from the real recipient, we can identify and thus drop the bad aggregates more accurately.

3) Aggregation: To make the rate-limiting more effective, it is better to consider the aggregates with the similar percentage of bad packets together. This may need to combine or separate aggregates dynamically. Moreover, it may be more costeffective to consider the aggregation of small aggregates as a whole. An alternative is to ignore the small aggregate for now and consider it later at some downstream domains when it has converged to a big enough aggregate. We plan to study more about dynamic aggregation and the impacts of these two different strategies on small aggregates in the future.

4) Simulation: In the topology shown in Fig. 11, $s_{0}$ is a DoS attacker and $s_{1}$ is a good user. In order to simplify the problem, we assume $d_{0}$ and $d_{1}$ have a way to identify the attack packets, such as based on the source IP address. We attach a query agent to $r_{0}$, and answer agents to $d_{0}$ and $d_{1}$. The traffic arriving at the queue $\left\langle r_{0}, r_{1}\right\rangle$ is separated into aggregates based on the destination IP address, $d_{0}$ and $d_{1}$. In our NS-2 simulation, the bandwidth of $\left\langle r_{0}, r_{1}\right\rangle$ is $0.81 \mathrm{Mbps}$, the probability to generate a query is 0.005 and the time period to estimate the percentage of bad packets is 15 seconds.

Table V shows the background UDP traffic in the simulation. Besides, we also set up eight TCP(FTP) sessions between $s_{1}$ and $d_{0}$. Different from the UDP traffic, these good TCP sessions start at 0.0 and end at 305.0.

We run the simulation when the type of $\left\langle r_{0}, r_{1}\right\rangle$ is I4 or Droptail or RED (Random Early Dropping) during the DoS attack. Fig. 13 shows the total throughput of eight TCP 
TABLE V

THE SETTING OF BACKGROUND TRAFFIC

\begin{tabular}{|c|c|c|c|c|c|}
\hline src & dst & $\begin{array}{c}\text { bad traffic } \\
\text { volume }(\mathrm{Mbps})\end{array}$ & $\begin{array}{c}\text { total traffic } \\
\text { volume }(\mathrm{Mbps})\end{array}$ & $\begin{array}{c}\text { start } \\
\text { (Seconds) }\end{array}$ & $\begin{array}{c}\text { end } \\
\text { (Seconds) }\end{array}$ \\
\hline$s_{0}$ & $d_{0}$ & 0.1 & 0.1 & 5.0 & 310.0 \\
$s_{0}$ & $d_{1}$ & 0.3 & 0.3 & 5.0 & 310.0 \\
$s_{1}$ & $d_{0}$ & 0 & 0.3 & 0 & 310.0 \\
$s_{1}$ & $d_{1}$ & 0 & 0.1 & 0 & 310.0 \\
\hline
\end{tabular}

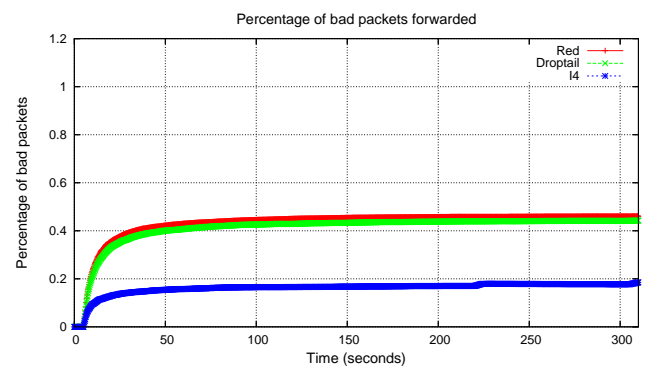

Fig. 12. Percentage of bad packets forwarded by various types of queues

sessions to $d_{0}$ averaged every 25 seconds with each type of queue. Fig. 12 shows the percentage of bad packets forwarded by each type of queue. The simulation results demonstrate a seven fold improvement in TCP throughput and a four-fold reduction in the percentage of bad packets.

\section{AdVAntages of I4}

\section{A. Incentive of support}

With I4, the participating domains could enjoy valuable information that complements their local knowledge and provides a more comprehensive view of the Internet activities. This collaboration mode has proven to be more effective than doing-it-alone mode. For example, in the DoS attack, not only the customer domain can avoid the saturation of its link, but also the ISP domain can reduce its network traffic load and serve its customer better. We believe that there are mutual benefits, thus strong incentives, for domains to collaborate together by deploying I4.

\section{B. Minimal changes}

Built on the top of the current routing infrastructure and TCP/IP stack, I4 makes minimal changes to the current Internet infrastructure. This avoids disrupting the current Internet service and interfering with the operations of legacy nodes or non-participants, thus shortening the time of transition.

\section{Incremental deployment}

I4 can be deployed incrementally. Even when only a small number of ISPs deploy I4 in the current power-law Internet, a large portion of unwanted traffic could be filtered out. Reference [9] shows that 50 ASes with the highest node degrees could cover approximately $90 \%$ of all the paths and thus are able to examine most of the Internet traffic. Deploying I4 in these big ASes can also provide scalability because an end domain does not have to communicate with many other domains. In summary, both the ISPs and the customer domains

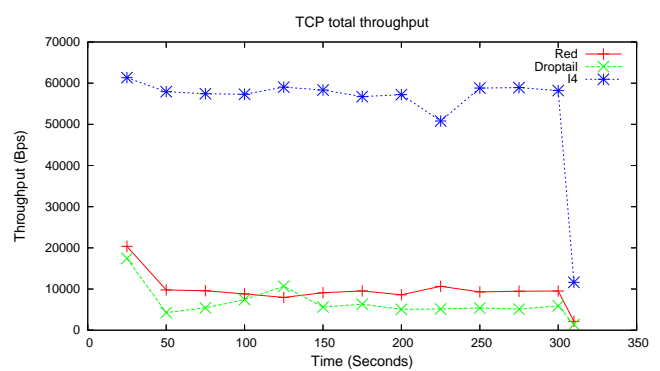

Fig. 13. Throughput of TCP sessions with RED, Droptail and I4 queuing

can enjoy immediate benefits even with a small number of deployments, which in return attracts more and more domains to participate.

\section{Efficiency}

The information exchange procedure is stateless, efficient and robust against the state or resource exhaustion attack and the unexpected situations, such as packet loss, reordering etc. Considering the burdensome recovery costs, the design of I4 makes a good choice in the tradeoff between reliability and efficiency.

\section{E. Scalability}

By designating one or several agents responsible for the task of information interaction of the whole domain, the number of nodes to be upgraded in the Internet remains minimal; thus this kind of hierarchy organization provides scalability ${ }^{11}$. Moreover, the routers can aggregate the flows, e.g. based on the network prefix, thus the amount of information may scale well even with large number of network flows.

\section{F. Universal}

I4 is a universal architecture that can help tackle a large range of problems. For example, during worm outbreak, the capability of intrusion and anomaly detection in one customer domain can discover the worm signature. With I4, this knowledge can be further distributed to other domains; thus the spread of worm can be stopped much faster than before. Also the victim domain could generate the signature of DDoS attack traffic if possible and distribute this knowledge to its upstream domain to throttle the attack. Even when the signature is not available, as we show before, with the information of just one packet accumulated together, i.e. whether this packet received by the victim is "good" or "bad", the ISP domain can preferentially drop attack packets and thus save more good packets.

I4 can also help with the network diagnosis. Today only a few $e 2 e$ measurement tools, such as traceroute and ping, and perhaps BGP information are available to help detect and diagnose the network problem, which provides limited and sometimes confusing information. With I4, the ISP domain

\footnotetext{
${ }^{11}$ If each router performs the functionality of I4 agent, the communication delay can be reduced. So there is a tradeoff between scalability and performance.
} 
can provide the information, such as the link condition, the statistic of flows, the root-cause of network failure, to the customer domain so that the customer domain can recover from the diaster or leverage this information to improve the $e 2 e$ performance, such as by multi-path routing, source routing etc.

\section{DISCUSSION}

\section{A. Security analysis}

The attacker may try to evade, disable or even attack I4. In the following, we discuss related threats and countermeasures.

To eavesdrop, modify, intercept and even drop the I4 packet, the attacker must attach to the same routing paths taken by these packets. This prerequisite raises the bar to launch this kind of attack and also limits the scope of attackers to be at certain locations. The communicating peers can establish the security association (SA) to protect the confidentiality and integrity of information exchanged. In the future we plan to study how to establish SA under the practical constraints. Note that even with SA a Man-in-Middle can still drop the I4 packet. The "WATCHER" method [20] can be used to detect disruptive routers.

The attacker may try to flood the customer domain with forged queries. If the attacker is inside an I4 domain, the security association between the border router and the I4 agent can detect the forged I4 packet. If an attacker is inside a regular domain or an I4 domain is compromised, the query flood can be injected into the Internet. This issue can be addressed by rate-limiting the queries at border routers. For example, for a certain aggregate, if the ratio between the number of queries and the number of data packets is significantly larger than a threshold (e.g. the query generation probability), the extra queries will be dropped. Although the query generated by a good domain can be dropped, the forged query actually makes the system more effective because it exactly catches an attack packet. Also the impacts on the percentage estimation should be little as the algorithm works well with a small number of queries. Another approach is to identify the domains where excess queries come from and then inform other benign I4 domains to block I4 packets from those domains. This would further motivate the local investigation.

The attackers may try to overwhelm the ISP domains by flooding with answer messages. As the forged answer messages do not contain the valid cookies generated by a sequence of I4 domains, they will be dropped when arriving at the first I4 domain, which significantly saves the network bandwidth.

A greedy/malicious customer domain may try to achieve more benefits by providing wrong answers. For example, it may identify an "attack" packet as "good" intentionally so that the probability for its aggregate to be dropped is smaller. However it ends up with receiving more unwanted packets, which wastes its resources and adversely affects the performance of its legitimate users. Furthermore, in order to incent an honest answer, the ISP domain can provide differentiated services to aggregates. For example, the aggregate containing a higher percentage of bad packets is assigned a larger probability of query generation. Thus the percentage estimation is more accurate and the changes can be detected more quickly. Note that the total number of queries generated is still kept the same. Thus the incentive to provide an honest answer is increased. In the future we plan to apply game theory to analyze the interaction of different answer strategies.

Last but not least, our information exchange procedure provides anonymity and recoverable privacy, as the identity of original I4 domain could be encrypted during the transmission. This would further increase the incentive of participation, especially when the ISP domain may concern that the information provided becomes the evidence against itself later.

\section{B. Availability of 14 under stress}

The availability of I4 service is important to resist unwanted traffic in the Internet. If there is more unwanted traffic, the I4 agent more likely generates an effective query, which in return helps remove the unwanted traffic. In other words, I4 is self-reinforcing and self-protecting. Also a step-by-step approach is possible: Firstly, the victim domain informs the upstream domain of the acceptable amount of traffic when a severe DDoS attack is detected, as in [10]. After the upstream domains stop forwarding the excess traffic, the victim domain provides more answer messages to help the upstream I4 domain drop more "bad" packets. We plan to conduct further experiments in the test bed to evaluate these ideas.

\section{RELATED WORKS}

Our work leverages on many previous works in the literature. Due to the limitation of space, we focus on the DDoS related works.

Early works in this field primarily target at the spoofing DDoS attack. Ingress filtering [13] prevents this attack by checking whether the source IP address falls into the network prefix of an edge domain. However there is no strong incentive of deployment because 1) the effectiveness of such mechanisms depends on universal deployment; 2) the attacker is able to evade this mechanisms with just minor efforts. iTrace/traceback [1] [2] [3] [4] [5] is proposed to traceback the true origin of spoofed packets by providing additional (path) information to the victim. Despite a significant step, it fails to consider the incentives of deployment: the information provided to victims cannot help stop the unwanted traffic remotely injected into the Internet. Given that the legal actions may take a long time to start, ISPs and victims do not see the immediate benefits to justify the cost of deploying iTrace/traceback. References [7] and [16] proposed to help filter the spoofed packet based on either the embedded path informaiton or "hop count" at the edge of domains. However, the attack traffic cannot be dropped early.

ACC/Pushback [11] proposed to rate-limit the high-volume aggregates during link congestion and to further push such information back to upstream routers. In [10], a server under stress installs router throttles in the upstream routers so that excess traffic is dropped before arriving. However neither can 
distinguish the legitimate traffic from the attack traffic. I4 can be combined with them to drop more attack packets.

SIFF [8] and TVA [17] proposed the concept of "capability" that allows an end host to selectively drop the unwanted packets. Our proposal can provide the same functions as them. The main differences are as follows: 1) SIFF and TVA implicitly assigns a lower priority to a current flow by not renewing the previous capability. Instead, we explicitly send the feedback in a statistically generated answer message so that the information of individual packets can be accumulated into the knowledge of aggregates. 2) With undirectional traffic and short flows, both SIFF and TVA are less efficient; moreover TVA has to adjust the bandwidth for the initial requests based on different types of traffic. Our mechanism utilizes "trend" in the traffic and the extracted knowledge to preferentially drop the attack packets in any kind of traffic. 3) TVA used "fairqueuing" to further prevent the flood of initial requests. Our mechanism assigns the different priorities to different initial request aggregates, so that more requests from attackers are dropped. In fact, our proposal can be combined with TVA to achieve both fairness and prioritization. For example, we can reserve some bandwidth for each flow and allocate the rest based on priorities.

Furthermore, there are a lot of works on analyzing and detecting the DDoS traffic based on statistical methods, such as [6] [15]. Reference [14] reports the DoS attack prevalence and dynamics in the Internet. These works greatly help us understand the DDoS attack.

\section{CONClusions}

We have presented the design of I4, a network infrastructure of information interaction in the Internet. We demonstrate the advantages of I4 in the case of DDoS attacks. With I4, the customer domain expresses its preferences about the current flows to the ISP domain so that the unwanted traffic can be dropped early. We develop algorithms to tackle the practical challenges related to the information exchange and knowledge learning. Our simulation results and theoretical analyses show that the performance can be greatly improved with the information exchanged. Compared with previous works, our proposal can handle many different types of unwanted traffic. The design of I4 also makes it easy to bear to practice.

\section{ACKNOWLEDGE}

This work was supported in part by NSF award \# 0520333. Professor V. Rao Vemuri is supported by AFOSR (Grant FA9550-04-1-0159).

\section{REFERENCES}

[1] S. M. Bellovin, "ICMP Traceback Messages", Internet Draft, March 2000.

[2] S. Savage, D. Wetherall, A. Karlin, and T. Anderson, "Practical Network Support for IP Traceback", Proceedings of ACM SIGCOMM, August 2000.

[3] A. C. Snoeren, C. Partridge, L. A. Sanchez, C. E. Jones, F. Tchakountio, S. T. Kent, and W. T. Strayer, "Hash-Based IP Traceback", Proceedings of ACM SIGCOMM, August 2001.

[4] D. Song, and Adrian Perrig, "Advanced and Authenticated Marking Schemes for IP Traceback”, Proceedings of IEEE INFOCOM, April 2001.
[5] A. Mankin, D. Massey, C.-L. Wu, S. F. Wu, and L. Zhang, "On Design and Evaluation of Intention-Driven ICMP Traceback", Proceedings of IEEE International Conference on Computer Communications and Networks, Octobor 2001.

[6] A. Hussain, J. Heidemann, and C. Papadopoulos, "A Framework for Classifying Denial of Service Attacks", Proceddings of ACM SIGCOMM, August 2003.

[7] A. Yaar, A. Perrig, and D. Song, "Pi: A Path Identification Mechanism to Defend against DDoS Attacks", Proceedings of IEEE Symposium on Security and Privacy, May 2003.

[8] A. Yaar, A. Perrig, and D. Song, "SIFF: A Stateless Internet Flow Filter to Mitigate DDoS Flooding Attacks", Proceedings of IEEE Symposium on Security and Privacy, May 2004.

[9] Y. Xie, V. Sekar, D. Maltz, M. Reiter, and H. Zhang, "Worm Origin Identification Using Random Moonwalks", Proceedings of IEEE Symposium on Security and Privacy, May 2005.

[10] D. Yau, J. Lui, F. Liang, and Y. Yam, "Defending against distributed denial-of-service attacks with max-min fair server-centric router throttles", IEEE/ACM Transactions on Networking, Volume 13, Issue 1 (February 2005), Pages: 29 - 42, Year of Publication: 2005, ISSN:10636692.

[11] R. Mahajan, S. M. Bellovin, S. Floyd, J. Ioannidis, V. Paxson, and S. Shenker, "Controlling High Bandwidth Aggregates in the Network" (Extended Version), July 2001.

[12] J. Ioannidis, and S. M. Bellovin, "Implementing Pushback: Router-Based Defense Against DDoS Attacks", Proceedings of Network and Distributed System Security Symposium, February 2002.

[13] P. Ferguson, and D. Senie, "Network Ingress Filtering: Defeating Denial of Service Attacks Which Employ IP Source Address Spoofing", RFC 2267, January 1998.

[14] D. Moore, G. M. Voelker, and S. Savage, "Inferring Internet Denial-ofService Activity", Proceedings of USENIX Security Symposium, August 2001.

[15] H. Wang, D. Zhang, and K. G. Shin, "Detecting SYN Flooding Attacks", Proceedings of IEEE INFOCOM, 2002.

[16] C. Jin, H. Wang, and K. G. Shin, "Hop-Count Filtering: An Effective Defense Against Spoofed Traffic", Proceedings of ACM CCS, October 2003.

[17] X. Yang, D. Wetherall, and T. Anderson, "A DoS-limiting Network Architecture", Proceedings of ACM SIGCOMM, August 2005.

[18] http://nms.lcs.mit.edu/activeware/

[19] http://i3.cs.berkeley.edu/

[20] K. A. Bradley, S. Cheung, N. Puketza, B. Mukherjee, and R. A. Olsson, "Detecting disruptive routers: A distributed network monitoring approach", Proceedings of IEEE Symposium on Security and Privacy, May 1998. 